PROCEEDINGS OF THE

AMERICAN MATHEMATICAL SOCIETY

Volume 138, Number 7, July 2010, Pages 2493-2503

S 0002-9939(10)10274-3

Article electronically published on February 24, 2010

\title{
INTEGRAL CONDITIONS ON THE UNIFORM ASYMPTOTIC STABILITY FOR TWO-DIMENSIONAL LINEAR SYSTEMS WITH TIME-VARYING COEFFICIENTS
}

\author{
JITSURO SUGIE AND MASAKAZU ONITSUKA
}

(Communicated by Yingfei Yi)

\begin{abstract}
This paper is concerned with the uniform asymptotic stability of the zero solution of the linear system $\mathbf{x}^{\prime}=A(t) \mathbf{x}$ with $A(t)$ being a $2 \times 2$ matrix. Our result can be used without knowledge about a fundamental matrix of the system.
\end{abstract}

\section{INTRODUCTION}

We consider the linear system

$$
\mathbf{x}^{\prime}=A(t) \mathbf{x}=\left(\begin{array}{cc}
-e(t) & f(t) \\
-g(t) & -h(t)
\end{array}\right) \mathbf{x}
$$

where the prime denotes $d / d t$; the coefficients $e(t), f(t), g(t)$ and $h(t)$ are continuous for $t \geq 0$, and they are allowed to change sign. It is clear that system (10) has the zero solution $(x(t), y(t)) \equiv(0,0)$.

In the case where $e(t) \equiv h(t)$ and $f(t) \equiv g(t)$, a fundamental matrix $X(t)$ for system (10) is given by

$$
X(t)=\left(\begin{array}{cc}
\cos G(t) & \sin G(t) \\
-\sin G(t) & \cos G(t)
\end{array}\right) \exp (-H(t)),
$$

where

$$
G(t)=\int_{0}^{t} g(\tau) d \tau \quad \text { and } \quad H(t)=\int_{0}^{t} h(\tau) d \tau .
$$

Let $\|\mathbf{x}\|$ be the Euclidean norm of a vector $\mathbf{x}$. Then, we have

$$
\left\|X(t) X^{-1}(s)\right\| \stackrel{\text { def }}{=} \sup _{\|\mathbf{x}\|=1}\left\|X(t) X^{-1}(s) \mathbf{x}\right\|=\exp (-H(t)+H(s))
$$

for $0 \leq s \leq t<\infty$. Following Theorem 1 in the book [2, p. 54], in general, the zero solution of (1) is uniformly asymptotically stable (for the definition, see Section 2) if and only if there exist positive constants $R$ and $\rho$ such that

$$
\left\|X(t) X^{-1}(s)\right\| \leq R \exp (-\rho(t-s)) \quad \text { for } 0 \leq s \leq t<\infty .
$$

Received by the editors July 16, 2009, and, in revised form, October 30, 2009.

2010 Mathematics Subject Classification. Primary 34D05, 34D20; Secondary 34D23, 37C75.

Key words and phrases. Uniform asymptotic stability, linear systems, Coppel's criterion, Floquet theory, integrally positive.

(C)2010 American Mathematical Society Reverts to public domain 28 years from publication 
We therefore conclude that a necessary and sufficient condition for the zero solution of (11) to be uniformly asymptotically stable is that

$$
\int_{s}^{t} h(\tau) d \tau \geq \rho(t-s)-\sigma \quad \text { for } 0 \leq s \leq t<\infty
$$

with $\rho>0$ and $\sigma>0$ in the special case where $e(t) \equiv h(t)$ and $f(t) \equiv g(t)$.

For example, consider system (11) with

$$
e(t)=h(t)=0.1+\sin t \quad \text { and } \quad f(t)=g(t)=\frac{1}{2-\sin t} .
$$

Then

$$
\int_{s}^{t} h(\tau) d \tau=0.1(t-s)-\cos t+\cos s \geq 0.1(t-s)-2 .
$$

Hence, condition (4) is satisfied with $\rho=0.1$ and $\sigma=2$, and therefore the zero solution of (11) with (5) is uniformly asymptotically stable.

As another method, Floquet theory is available for this example, because all coefficients are periodic functions with period $2 \pi$. Note that $X(2 \pi)$ is the monodromy matrix of (1), where $X(t)$ is given in (2). Let $\lambda_{1}$ and $\lambda_{2}$ be the eigenvalues of the monodromy matrix $X(2 \pi)\left(\lambda_{1}\right.$ and $\lambda_{2}$ are often called the Floquet multipliers of (10). It follows from Floquet theory that the zero solution of (1) is uniformly asymptotically stable if and only if the Floquet multipliers $\lambda_{1}$ and $\lambda_{2}$ have magnitudes strictly less than 1. For example, Floquet theory can be found in the books [1, 3, 4, 6, 9.

Since

$$
G(t)=\int_{0}^{t} \frac{1}{2-\sin \tau} d \tau=\frac{2}{\sqrt{3}} \operatorname{Tan}^{-1}\left(\frac{2 \tan (t / 2)-1}{\sqrt{3}}\right)+\frac{2 m \pi}{\sqrt{3}}+\frac{\pi}{3 \sqrt{3}}
$$

for $m \pi<t \leq(m+1) \pi, m=0,1,2, \ldots$, and

$$
H(t)=\int_{0}^{t}(0.1+\sin \tau) d \tau=0.1 t+1-\cos t
$$

for $t>0$, it follows that $G(2 \pi)=2 \pi / \sqrt{3}$ and $H(2 \pi)=0.2 \pi$. Hence, from (2) it turns out that the monodromy matrix

$$
X(2 \pi)=\left(\begin{array}{cc}
\cos (2 \pi / \sqrt{3}) & \sin (2 \pi / \sqrt{3}) \\
-\sin (2 \pi / \sqrt{3}) & \cos (2 \pi / \sqrt{3})
\end{array}\right) e^{-0.2 \pi},
$$

and therefore the Floquet multipliers $\lambda_{1}$ and $\lambda_{2}$ are the roots of the equation

$$
\lambda^{2}-2 e^{-0.2 \pi} \cos \frac{2 \pi}{\sqrt{3}} \lambda+e^{-0.4 \pi}=0
$$

that is,

$$
\lambda_{1}=e^{-0.2 \pi}\left(\cos \frac{2 \pi}{\sqrt{3}}+i \sin \frac{2 \pi}{\sqrt{3}}\right) \quad \text { and } \quad \lambda_{2}=e^{-0.2 \pi}\left(\cos \frac{2 \pi}{\sqrt{3}}-i \sin \frac{2 \pi}{\sqrt{3}}\right) .
$$

Since the Floquet multipliers have modulus smaller than 1, the zero solution of (1) with (5) is uniformly asymptotically stable.

To confirm whether Coppel's criterion (3) is satisfied or not, of course, we need a fundamental matrix for system (11). Unfortunately, however, we cannot get a concrete expression of a fundamental matrix in the general case where $e(t) \not \equiv h(t)$ or $f(t) \not \equiv g(t)$. On the other hand, if the coefficients $e(t), f(t), g(t)$ and $h(t)$ are 
periodic, then, without knowledge of a fundamental matrix of (11), the Floquet multipliers $\lambda_{1}$ and $\lambda_{2}$ can be calculated by a numerical scheme. For example, consider system (11) with

$$
e(t)=0, \quad f(t)=g(t)=\frac{1}{2-\sin t} \quad \text { and } \quad h(t)=0.1+\sin t .
$$

Then, although we cannot find a fundamental matrix of (1), the Floquet multipliers $\lambda_{1}$ and $\lambda_{2}$ can be estimated as follows:

$$
\lambda_{1} \approx 0.1875612224300 \text { and } \lambda_{2} \approx 2.8443410859625>1 .
$$

Hence, the zero solution of (11) with (6) is not uniformly asymptotically stable.

The fault of Floquet theory is being unable to use it when some of the coefficients of (11) are not periodic. In this paper, we give sufficient conditions for the zero solution of (1) to be uniformly asymptotically stable, which are applicable even in cases where a fundamental matrix cannot be found and system (10) has non-periodic coefficients. In Section 2, we present the main result and give its proof. To illustrate our main result, we take some concrete examples.

\section{The MAIN RESUlT}

We denote the solution of (1) through $\left(t_{0}, \mathbf{x}_{0}\right) \in[0, \infty) \times \mathbb{R}^{2}$ by $\mathbf{x}\left(t ; t_{0}, \mathbf{x}_{0}\right)$. The zero solution of (1) is said to be uniformly stable if, for any $\varepsilon>0$, there exists a $\delta(\varepsilon)>0$ such that $t_{0} \geq 0$ and $\left\|\mathbf{x}_{0}\right\|<\delta$ imply $\left\|\mathbf{x}\left(t ; t_{0}, \mathbf{x}_{0}\right)\right\|<\varepsilon$ for all $t \geq t_{0}$. The zero solution is said to be uniformly attractive if there exists a $\delta_{0}>0$ such that, for every $\eta>0$, there is a $T(\eta)>0$ such that $t_{0} \geq 0$ and $\left\|\mathbf{x}_{0}\right\|<\delta_{0}$ imply $\left\|\mathbf{x}\left(t ; t_{0}, \mathbf{x}_{0}\right)\right\|<\eta$ for $t \geq t_{0}+T$. The zero solution is uniformly asymptotically stable if it is uniformly stable and is uniformly attractive. The most important point is that $\delta$ and $T$ can be chosen independent of $t_{0}$ in the definition of uniform asymptotic stability.

The concept of uniform asymptotic stability plays an essential role in perturbation problems. For example, if the zero solution of (1) is uniformly asymptotically stable and if $\mathbf{f}(t, \mathbf{x})$ and $\lambda(t)$ satisfy that $\|\mathbf{f}(t, \mathbf{x})\| \leq \lambda(t)\|\mathbf{x}\|$ for $t \geq 0$ and $\mathbf{x} \in \mathbb{R}^{2}$, where

$$
\int_{0}^{\infty} \lambda(s) d s<\infty
$$

then the zero solution of the perturbed system

$$
\mathbf{x}^{\prime}=A(t) \mathbf{x}+\mathbf{f}(t, \mathbf{x})
$$

is uniformly asymptotically stable. However, if $\delta$ and $T$ depend on $t_{0}$, then we cannot derive this conclusion. For the details, see [7] (also [1, pp. 169-170]). For this reason, the present study has a close relationship with perturbation problems.

Let

$$
\phi_{+}(t)=\max \{0, \phi(t)\} \quad \text { and } \quad \phi_{-}(t)=\max \{0,-\phi(t)\}
$$

for a continuous function $\phi(t)$. Then, it follows that $\phi(t)=\phi_{+}(t)-\phi_{-}(t)$ and $|\phi(t)|=\phi_{+}(t)+\phi_{-}(t)$. The function $\phi_{+}(t)$ is said to be integrally positive if

$$
\int_{I} \phi_{+}(t) d t=\infty
$$


for every set $I=\bigcup_{n=1}^{\infty}\left[\tau_{n}, \sigma_{n}\right]$ such that $\tau_{n}+\omega<\sigma_{n}<\tau_{n+1}$ for some $\omega>0$. For example, $\sin ^{2} t$ is an integrally positive function (see [5, 8]).

Throughout this paper, we assume that $f(t) g(t)>0$ and $g(t) / f(t)$ is differentiable for $t \geq 0$. Then, we may define

$$
\psi(t)=2 h(t)+\frac{f(t)}{g(t)}\left(\frac{g(t)}{f(t)}\right)^{\prime} .
$$

Our main result is as follows:

Theorem 1. Suppose that $f(t), g(t)$ and $h_{+}(t)$ are bounded for $t \geq 0$. Suppose also that

(i) $f(t) g(t)>0$ for $t \geq 0$ and $\liminf _{t \rightarrow \infty} f(t) g(t)>0$;

(ii) $\int_{0}^{\infty} e_{-}(t) d t<\infty, \int_{0}^{\infty} h_{-}(t) d t<\infty$ and $\int_{0}^{\infty} \psi_{-}(t) d t<\infty$;

(iii) $\psi_{+}(t)$ is integrally positive.

Then the zero solution of (1) is uniformly asymptotically stable.

Remark 1. As a paper related to Theorem 1, we can cite Hatvani [5]. Under the assumptions that $e(t) \geq 0, f(t)=g(t) \geq 0$ and $h(t) \geq 0$ for $t \geq 0$, he has given some sufficient conditions guaranteeing only asymptotic stability for system (1).

Before proving the main theorem, we present some values drawn from assumptions in Theorem 1. From assumption (i) and the boundedness of $f(t), g(t)$ and $h_{+}(t)$, we can choose positive numbers $\bar{f}, \underline{g}, \bar{h}, k$ and $K$ such that

$$
|f(t)| \leq \bar{f}, \quad \underline{g} \leq|g(t)|, \quad h_{+}(t) \leq \bar{h} \quad \text { and } \quad k \leq \frac{f(t)}{g(t)} \leq K
$$

for $t \geq 0$. We may assume that $k \leq 1 \leq K$. From assumption (ii), there exist positive constants $L$ and $M$ such that

$$
L=\int_{0}^{\infty}\left(2 e_{-}(s)+\psi_{-}(s)\right) d s \text { and } M=\int_{0}^{\infty} h_{-}(s) d s .
$$

It is known that $\psi_{+}(t)$ is integrally positive if and only if

$$
\liminf _{t \rightarrow \infty} \int_{t}^{t+\gamma} \psi_{+}(s) d s>0
$$

for every $\gamma>0$. Hence, there exist an $l>0$ and a $\hat{t}>0$ such that

$$
\int_{t}^{t+1} \psi_{+}(s) d s \geq l \text { for } t \geq \hat{t} .
$$

The above-mentioned values are used without notice in the proof of Theorem 1.

Proof of Theorem 1. We will prove the theorem by dividing it into seven steps.

Step 1. To prove the uniform stability of the zero solution of (1), for a given $\varepsilon>0$, we select

$$
\delta(\varepsilon)=\sqrt{\frac{k}{K e^{L}}} \varepsilon .
$$


Needless to say, $\delta<\varepsilon$. Let $t_{0} \geq 0$ and $\mathbf{x}_{0}=\left(x_{0}, y_{0}\right)$ be given. We will show that $t \geq t_{0}$ and $\left\|\mathbf{x}_{0}\right\|=\sqrt{x_{0}^{2}+y_{0}^{2}}<\delta$ imply $\left\|\mathbf{x}\left(t ; t_{0}, \mathbf{x}_{0}\right)\right\|<\varepsilon$. For convenience of notation, we write $\mathbf{x}(t)=\mathbf{x}\left(t ; t_{0}, \mathbf{x}_{0}\right)$ and $(x(t), y(t))=\mathbf{x}(t)$.

Let

$$
u(t)=\frac{f(t)}{g(t)} y^{2}(t) \quad \text { and } \quad v(t)=x^{2}(t)+u(t) .
$$

Then, $v(t) \geq x^{2}(t)+k y^{2}(t) \geq k\|\mathbf{x}(t)\|^{2}$ for $t \geq t_{0}$. Since

$$
v^{\prime}(t)=-2 e(t) x^{2}(t)-\psi(t) u(t) \leq\left(2 e_{-}(t)+\psi_{-}(t)\right) v(t)
$$

for $t \geq t_{0}$, we have

$$
\begin{aligned}
v(t) & \leq \exp \left(\int_{t_{0}}^{t}\left(2 e_{-}(s)+\psi_{-}(s)\right) d s\right) v\left(t_{0}\right) \leq e^{L} v\left(t_{0}\right) \\
& \leq e^{L} K\left(x_{0}^{2}+y_{0}^{2}\right)<K e^{L} \delta^{2}(\varepsilon)=k \varepsilon^{2}
\end{aligned}
$$

for $t \geq t_{0}$. Hence, we obtain

$$
\left\|\mathbf{x}\left(t ; t_{0}, \mathbf{x}_{0}\right)\right\|<\varepsilon \text { for } t \geq t_{0}
$$

and therefore, the zero solution of (1) is uniformly stable. This completes the proof of Step 1.

Hereafter, we will show that the zero solution of (10) is uniformly attractive.

Step 2. Let $\delta_{0}=1 / \sqrt{K e^{L}}$. For every $\eta>0$, a number $T(\eta)$ is decided as follows. To begin with, let

$$
\underline{v}=k \delta^{2}(\eta), \quad \mu=\min \left\{\frac{\underline{v}}{2}, \frac{k \underline{g}^{2} \underline{v}}{8 \bar{h}^{2}}\right\} \quad \text { and } \quad \tau=\hat{t}+\left[\frac{2(1+L)}{l \mu}\right]+2,
$$

where $\delta(\cdot)$ is the number given in (77) and $[c]$ means the greatest integer that is less than or equal to a real number $c$. Note that $\underline{v}, \mu$ and $\tau$ depend only on $\eta$. Consider the definite integral

$$
\int_{t}^{t+\mu \sqrt{k} /(8 \bar{f})} \psi_{+}(s) d s
$$

Then, the upper limit of integration depends only on $\eta$, and so does the integral. Let

$$
\nu=\liminf _{t \rightarrow \infty} \frac{1}{4} \int_{t}^{t+\mu \sqrt{k} /(8 \bar{f})} \psi_{+}(s) d s .
$$

Since $\psi_{+}(t)$ is integrally positive, the number $\nu$ is positive and depends only on $\eta$. From assumptions (ii) and (iii) it turns out that there exists a positive number $\sigma$ depending only on $\eta$ such that

$$
\int_{t}^{\infty}\left(2 e_{-}(s)+\psi_{-}(s)\right) d s \leq \min \left\{\frac{\mu}{4}, \frac{\mu \nu}{4}\right\}
$$

and

$$
\int_{t}^{t+\mu \sqrt{k} /(8 \bar{f})} \psi_{+}(s) d s \geq 2 \nu
$$

for $t \geq \sigma$, respectively. Using numbers $\mu, \nu, \sigma$ and $\tau$, we define

$$
T=\sigma+\left(\left[\frac{4}{\mu \nu}\right]+1\right)\left(\frac{3 e^{M}}{\bar{h}}+\tau\right) .
$$


Step 3 . Let $t_{0} \geq 0$ and let $\mathbf{x}_{0}=\left(x_{0}, y_{0}\right)$ be a point satisfying $\left\|\mathbf{x}_{0}\right\|=\sqrt{x_{0}^{2}+y_{0}^{2}}<\delta_{0}$. Consider a solution $\mathbf{x}(t)=\mathbf{x}\left(t ; t_{0}, \mathbf{x}_{0}\right)$ of (1) through $\left(t_{0}, \mathbf{x}_{0}\right)$. To prove the uniform attractivity of the zero solution of (1), it is enough to show that there exists a $t^{*} \in\left[t_{0}, t_{0}+T\right]$ such that

$$
\left\|\mathbf{x}\left(t^{*}\right)\right\|<\delta(\eta)
$$

In fact, because of Step 1, if (11) holds, then any solution $\mathbf{x}\left(t ; t^{*}, \mathbf{x}\left(t^{*}\right)\right)$ of (1) through $\left(t^{*}, \mathbf{x}\left(t^{*}\right)\right)$ satisfies that

$$
\left\|\mathbf{x}\left(t ; t^{*}, \mathbf{x}\left(t^{*}\right)\right)\right\|<\eta \quad \text { for } t \geq t^{*} .
$$

Since $t_{0}+T \geq t^{*}$, it follows that

$$
\left\|\mathbf{x}\left(t ; t_{0}, \mathbf{x}_{0}\right)\right\|<\eta \quad \text { for } t \geq t_{0}+T .
$$

There are two cases to consider: (a) $\eta \geq 1 / \sqrt{k}$ and (b) $0<\eta \leq 1 / \sqrt{k}$. In case (a), by (17), we have

$$
\delta_{0}=\frac{1}{\sqrt{K e^{L}}} \leq \sqrt{\frac{k}{K e^{L}}} \eta=\delta(\eta) .
$$

Hence, letting $t^{*}=t_{0}$, we obtain

$$
\left\|\mathbf{x}\left(t^{*}\right)\right\|=\left\|\mathbf{x}_{0}\right\|<\delta_{0} \leq \delta(\eta)
$$

namely, (11). This completes the proof. Thus, we have only to consider case (b) from now on. By way of contradiction, we will prove that inequality (11) holds. Suppose that

$$
\|\mathbf{x}(t)\| \geq \delta(\eta) \text { for } t_{0} \leq t \leq t_{0}+T
$$

Then, we have

$$
0<\underline{v}=k \delta^{2}(\eta) \leq k\|\mathbf{x}(t)\|^{2} \leq v(t)
$$

for $t_{0} \leq t \leq t_{0}+T$. Using (8) again, we get

$$
v(t) \leq e^{L} K\left(x_{0}^{2}+y_{0}^{2}\right)<K e^{L} \delta_{0}^{2}=1 \text { for } t \geq t_{0} .
$$

Step 4. If $u(t) \geq \mu / 2$ for any interval $\left[\alpha_{1}, \beta_{1}\right] \subset\left[t_{0}, t_{0}+T\right]$, then $\beta_{1}-\alpha_{1}<\tau$, where $\mu$ and $\tau$ are numbers given in Step 2. In fact, taking into account that

$$
\begin{aligned}
v^{\prime}(t) & =-2 e(t) x^{2}(t)-\psi(t) u(t) \\
& =-2 e(t) x^{2}(t)+\psi_{-}(t) u(t)-\psi_{+}(t) u(t)
\end{aligned}
$$

for $t \geq t_{0}$, from (13) we see that

$$
\begin{aligned}
0 \leq \psi_{+}(t) u(t) & =-v^{\prime}(t)-2 e(t) x^{2}(t)+\psi_{-}(t) u(t) \\
& \leq-v^{\prime}(t)+\left(2 e_{-}(t)+\psi_{-}(t)\right) v(t) \leq-v^{\prime}(t)+2 e_{-}(t)+\psi_{-}(t)
\end{aligned}
$$

for $t \geq t_{0}$. Integrating this inequality from $\alpha_{1}$ to $\beta_{1}$ and using (12) and (13), we obtain

$$
\begin{aligned}
\frac{\mu}{2} \int_{\alpha_{1}}^{\beta_{1}} \psi_{+}(s) d s & \leq \int_{\alpha_{1}}^{\beta_{1}} \psi_{+}(s) u(s) d s \leq-\int_{\alpha_{1}}^{\beta_{1}} v^{\prime}(s) d s+\int_{\alpha_{1}}^{\beta_{1}}\left(2 e_{-}(s)+\psi_{-}(s)\right) d s \\
& \leq v\left(\alpha_{1}\right)-v\left(\beta_{1}\right)+L<1+L
\end{aligned}
$$

Let

$$
m=\left[\frac{2(1+L)}{l \mu}\right]+1 .
$$


Taking $m \geq 2(1+L) /(l \mu)$ into account, we see that

$$
\begin{aligned}
\int_{t}^{t+m} \psi_{+}(s) d s & =\int_{t}^{t+1} \psi_{+}(s) d s+\int_{t+1}^{t+2} \psi_{+}(s) d s+\cdots+\int_{t+m-1}^{t+m} \psi_{+}(s) d s \\
& \geq l m \geq \frac{2(1+L)}{\mu}
\end{aligned}
$$

for $t \geq \hat{t}$. If $\alpha_{1} \geq \hat{t}$, then by (15) we have

$$
\int_{\alpha_{1}}^{\beta_{1}} \psi_{+}(s) d s \leq \frac{2(1+L)}{\mu} \leq \int_{\alpha_{1}}^{\alpha_{1}+m} \psi_{+}(s) d s,
$$

and therefore $\beta_{1}-\alpha_{1} \leq m<\tau$. If $\alpha_{1}<\hat{t}$, then by (15) we have

$$
\int_{\alpha_{1}}^{\beta_{1}} \psi_{+}(s) d s \leq \frac{2(1+L)}{\mu} \leq \int_{\hat{t}}^{\hat{t}+m} \psi_{+}(s) d s \leq \int_{\alpha_{1}}^{\alpha_{1}+\hat{t}+m} \psi_{+}(s) d s .
$$

Hence, $\beta_{1}-\alpha_{1} \leq \hat{t}+m<\tau$. Thus, it turns out that the beginning sentence of Step 4 is true.

Step 5. If $u(t) \leq \mu$ for any interval $\left[\alpha_{2}, \beta_{2}\right] \subset\left[t_{0}, t_{0}+T\right]$, then $\beta_{2}-\alpha_{2} \leq 2 e^{M} / \bar{h}$. In fact, since

$$
u(t)=\frac{f(t)}{g(t)} y^{2}(t), \quad v(t)=x^{2}(t)+u(t) \quad \text { and } \quad \mu=\min \left\{\frac{\underline{v}}{2}, \frac{k \underline{g}^{2} \underline{v}}{8 \bar{h}^{2}}\right\},
$$

we see that

$$
|x(t)|=\sqrt{v(t)-u(t)} \geq \sqrt{\underline{v}-\mu} \geq \sqrt{\frac{\underline{v}}{2}}
$$

and

$$
|y(t)|=\sqrt{\frac{g(t)}{f(t)} u(t)} \leq \sqrt{\frac{\mu}{k}} \leq \frac{\underline{g}}{2 \bar{h}} \sqrt{\frac{\underline{v}}{2}}
$$

for $\alpha_{2} \leq t \leq \beta_{2}$. Note that

$$
y^{\prime}(t)-h_{-}(t) y(t)=-g(t) x(t)-h_{+}(t) y(t)
$$

for $t \geq t_{0}$. Then, using (16) and (17), we obtain

$$
\begin{aligned}
\left|\left(\exp \left(-\int_{t_{0}}^{t} h_{-}(s) d s\right) y(t)\right)^{\prime}\right| & \geq \exp \left(-\int_{t_{0}}^{t} h_{-}(s) d s\right)\left(|g(t)||x(t)|-h_{+}(t)|y(t)|\right) \\
& \geq \frac{g e^{-M}}{2} \sqrt{\frac{\underline{v}}{2}}>0
\end{aligned}
$$


for $\alpha_{2} \leq t \leq \beta_{2}$. Hence, combining this with (17), we get

$$
\begin{aligned}
\frac{\underline{\underline{h}} \sqrt{\frac{\underline{v}}{2}}}{} & \geq\left|y\left(\beta_{2}\right)\right|+\left|y\left(\alpha_{2}\right)\right| \\
& \geq\left|\exp \left(-\int_{t_{0}}^{\beta_{2}} h_{-}(s) d s\right) y\left(\beta_{2}\right)-\exp \left(-\int_{t_{0}}^{\alpha_{2}} h_{-}(s) d s\right) y\left(\alpha_{2}\right)\right| \\
& =\left|\int_{\alpha_{2}}^{\beta_{2}}\left(\exp \left(-\int_{t_{0}}^{t} h_{-}(s) d s\right) y(t)\right)^{\prime} d t\right| \\
& =\int_{\alpha_{2}}^{\beta_{2}}\left|\left(\exp \left(-\int_{t_{0}}^{t} h_{-}(s) d s\right) y(t)\right)^{\prime}\right| d t \geq \frac{\underline{g} e^{-M}}{2} \sqrt{\frac{\underline{v}}{2}}\left(\beta_{2}-\alpha_{2}\right),
\end{aligned}
$$

and therefore $\beta_{2}-\alpha_{2} \leq 2 e^{M} / \bar{h}$. Thus, it turns out that the beginning sentence of Step 5 is true.

Step 6. Let

$$
J_{i}=\left[t_{0}+\sigma+(i-1)\left(\frac{3 e^{M}}{\bar{h}}+\tau\right), t_{0}+\sigma+i\left(\frac{3 e^{M}}{\bar{h}}+\tau\right)\right]
$$

for any $i \in \mathbb{N}$. Then, for each $i \in \mathbb{N}$, the length of $J_{i}$ is $3 e^{M} / \bar{h}+\tau$. We can divide the interval $\left[t_{0}+\sigma, t_{0}+T\right]$ as follows:

$$
\left[t_{0}+\sigma, t_{0}+T\right]=J_{1} \cup J_{2} \cup \cdots \cup J_{[4 /(\mu \nu)]+1} .
$$

Let us examine the motion of $u(t)$ in the interval $J_{1}$. It turns out that there exists a $t_{1} \in\left[t_{0}+\sigma, t_{0}+\sigma+\tau\right] \subset J_{1}$ such that $u\left(t_{1}\right)<\mu / 2$. In fact, if $u(t) \geq \mu / 2$ for $t \in\left[t_{0}+\sigma, t_{0}+\sigma+\tau\right] \subset\left[t_{0}, t_{0}+T\right]$, then by the conclusion of Step 4 , we have

$$
\tau=t_{0}+\sigma+\tau-\left(t_{0}+\sigma\right)=\beta_{1}-\alpha_{1}<\tau .
$$

This is a contradiction. It also turns out that there exists a $t_{2} \in\left[t_{0}+\sigma+\tau, t_{0}+\right.$ $\left.\sigma+3 e^{M} / \bar{h}+\tau\right] \subset J_{1}$ such that $u\left(t_{2}\right)>\mu$. In fact, if $u(t) \leq \mu$ for $t \in\left[t_{0}+\sigma+\tau, t_{0}+\right.$ $\left.\sigma+3 e^{M} / \bar{h}+\tau\right] \subset\left[t_{0}, t_{0}+T\right]$, then by the conclusion of Step 5, we have

$$
\frac{3 e^{M}}{\bar{h}}=t_{0}+\sigma+\frac{3 e^{M}}{\bar{h}}+\tau-\left(t_{0}+\sigma+\tau\right)=\beta_{2}-\alpha_{2} \leq \frac{2 e^{M}}{\bar{h}} .
$$

This is a contradiction. Hence, because of the continuity of $u(t)$, there exists an interval $[\alpha, \beta] \subset\left[t_{1}, t_{2}\right]$ such that $u(\alpha)=\mu / 2, u(\beta)=\mu$ and

$$
\frac{\mu}{2} \leq u(t) \leq \mu \text { for } \alpha \leq t \leq \beta .
$$

Hence, by (9) and (13) we have

$$
\begin{aligned}
\frac{\mu}{2} & =u(\beta)-u(\alpha)=\int_{\alpha}^{\beta} u^{\prime}(s) d s=\int_{\alpha}^{\beta}(-\psi(s) u(s)-2 f(s) x(s) y(s)) d s \\
& \leq \int_{\alpha}^{\beta}\left(\psi_{-}(s) v(s)+2|f(s) x(s) y(s)|\right) d s \leq \frac{\mu}{4}+2 \bar{f} \int_{\alpha}^{\beta}|x(s) y(s)| d s .
\end{aligned}
$$

Consequently,

$$
\frac{\mu}{8 \bar{f}} \leq \int_{\alpha}^{\beta}|x(s) y(s)| d s .
$$


Using (13) again, we can estimate that

$$
|x(t)|=\sqrt{v(t)-u(t)}<1 \quad \text { and }|y(t)|=\sqrt{\frac{g(t)}{f(t)} u(t)} \leq \sqrt{\frac{v(t)}{k}}<\frac{1}{\sqrt{k}}
$$

for $t \geq t_{0}$. We therefore conclude that

$$
\frac{\mu \sqrt{k}}{8 \bar{f}}<\beta-\alpha .
$$

Step 7. From the conclusion of Step 6 with (9), (10), (14) and (18) it turns out that

$$
\begin{aligned}
\mu \nu & \leq \frac{\mu}{2} \int_{\alpha}^{\alpha+\mu \sqrt{k} /(8 \bar{f})} \psi_{+}(s) d s \leq \frac{\mu}{2} \int_{\alpha}^{\beta} \psi_{+}(s) d s \\
& \leq \int_{\alpha}^{\beta} \psi_{+}(s) u(s) d s \leq \int_{\alpha}^{\beta}\left(-v^{\prime}(s)+2 e_{-}(s)+\psi_{-}(s)\right) d s \\
& =v(\alpha)-v(\beta)+\int_{\alpha}^{\beta}\left(2 e_{-}(s)+\psi_{-}(s)\right) d s \leq v(\alpha)-v(\beta)+\frac{\mu \nu}{4} .
\end{aligned}
$$

Hence, we have

$$
v(\beta)-v(\alpha) \leq-\frac{3 \mu \nu}{4} .
$$

Using (9) and (14) again, we get

$$
v(\alpha)-v\left(t_{0}+\sigma\right)=\int_{t_{0}+\sigma}^{\alpha} v^{\prime}(s) d s \leq \int_{t_{0}+\sigma}^{\alpha}\left(2 e_{-}(s)+\psi_{-}(s)\right) d s \leq \frac{\mu \nu}{4}
$$

and

$$
\begin{aligned}
v\left(t_{0}+\sigma+\frac{3 e^{M}}{\bar{h}}+\tau\right)-v(\beta) & =\int_{\beta}^{t_{0}+\sigma+3 e^{M} / \bar{h}+\tau} v^{\prime}(s) d s \\
& \leq \int_{\beta}^{t_{0}+\sigma+3 e^{M} / \bar{h}+\tau}\left(2 e_{-}(s)+\psi_{-}(s)\right) d s \leq \frac{\mu \nu}{4} .
\end{aligned}
$$

We therefore conclude that

$$
\begin{aligned}
\int_{J_{1}} v^{\prime}(s) d s & =v\left(t_{0}+\sigma+\frac{3 e^{M}}{\bar{h}}+\tau\right)-v(\beta)+v(\beta)-v(\alpha)+v(\alpha)-v\left(t_{0}+\sigma\right) \\
& \leq \frac{\mu \nu}{4}-\frac{3 \mu \nu}{4}+\frac{\mu \nu}{4}=-\frac{\mu \nu}{4} .
\end{aligned}
$$

By means of the same process as in the proof of Steps 6 and 7, we see that

$$
\int_{J_{i}} v^{\prime}(s) d s \leq-\frac{\mu \nu}{4} \quad \text { for } 1 \leq i \leq[4 /(\mu \nu)]+1,
$$

and therefore

$$
v\left(t_{0}+T\right)-v\left(t_{0}+\sigma\right)=\sum_{i=1}^{[4 /(\mu \nu)]+1} \int_{J_{i}} v^{\prime}(s) d s \leq-\frac{\mu \nu}{4}\left(\left[\frac{4}{\mu \nu}\right]+1\right)<-1 .
$$

Hence, from (13) it follows that

$$
v\left(t_{0}+T\right)<v\left(t_{0}+\sigma\right)-1<0 .
$$

This contradicts the fact that $v(t) \geq 0$ for $t \geq t_{0}$. Thus, in case (b) as well as in case (a), inequality (11) holds. The proof of Theorem 1 is thus complete. 


\section{EXAMPLES}

We illustrate our main result with simple examples to which Coppel's criterion and Floquet theory cannot be applied. To present examples, we define a function as follows: let $r$ be a number satisfying $0<r<1$ and let

$$
p(t)= \begin{cases}\frac{t}{2-r^{n}}+2(n-1)\left(1-\frac{1}{2-r^{n}}\right) & \text { if } 2(n-1) \leq t<2 n-r^{n}, \\ \frac{t}{r^{n}}+2 n\left(1-\frac{1}{r^{n}}\right) & \text { if } 2 n-r^{n} \leq t<2 n\end{cases}
$$

for any $n \in \mathbb{N}$. It is clear that the graph of $p(t)$ is a broken line (see Figure 1(a) below). As shown in Figure 1(b) below, the composite function $\sin (p(t) \pi)$ changes sign, but it is not periodic and not even almost periodic. It is easy to check that $\max \{0, \sin (p(t) \pi)\}$ is an integrally positive function and $\max \{0,-\sin (p(t) \pi)\}$ is an integrable function.
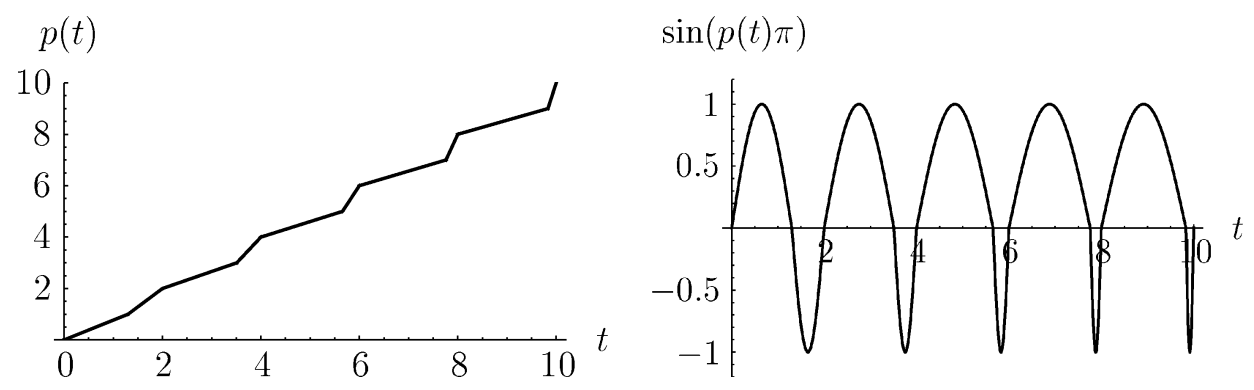

FiguRE 1. (a) The graph of $p(t)$ with $r=0.7$; (b) the graph of $\sin (p(t) \pi)$ with $r=0.7$

Example 1. Consider system (1) with

$$
e(t)=0, \quad f(t)=g(t)=1 \quad \text { and } \quad h(t)=\sin (p(t) \pi) .
$$

Then the zero solution is uniformly asymptotically stable.

It is clear that $f(t), g(t)$ and $h_{+}(t)$ are bounded and $g(t) / f(t)$ is differentiable for $t \geq 0$, and assumption (i) is satisfied. Assumptions (ii) and (iii) are also satisfied. In fact, taking $\psi(t)=2 h(t)$ into account, we see that

$$
\int_{0}^{\infty} e_{-}(t) d t=0, \quad \int_{0}^{\infty} h_{-}(t) d t<\sum_{i=1}^{\infty} r^{i}=\frac{r}{1-r}, \quad \int_{0}^{\infty} \psi_{-}(t) d t<\frac{2 r}{1-r}
$$

and $\psi_{+}(t)$ is integrally positive. Thus, by virtue of Theorem 1 , we conclude that the zero solution is uniformly asymptotically stable.

Example 2. Consider system (1) with

$$
e(t)=h(t)=\sin (p(t) \pi), \quad f(t)=1 \quad \text { and } \quad g(t)=\frac{1+t}{2+t} .
$$

Then the zero solution is uniformly asymptotically stable. 
Since $\psi(t)=2 h(t)+1 /((1+t)(2+t))$, it turns out that

$$
\psi_{+}(t)>2 h_{+}(t) \text { and } \psi_{-}(t)<2 h_{-}(t)
$$

for $t \geq 0$. Hence, it is easy to confirm that all of the assumptions in Theorem 1 are satisfied. We omit the details.

\section{REFERENCES}

[1] F. Brauer and J. Nohel, The Qualitative Theory of Ordinary Differential Equations, W. A. Benjamin, New York-Amsterdam, 1969; (revised) Dover, New York, 1989.

[2] W. A. Coppel, Stability and Asymptotic Behavior of Differential Equations, Heath, Boston, 1965. MR0190463 (32:7875)

[3] J. Cronin, Differential Equations: Introduction and Qualitative Theory, 2nd ed., Monographs and Textbooks in Pure and Applied Mathematics, 180, Marcel Dekker, New York-Basel-Hong Kong, 1994. MR:1275827 (95b:34001)

[4] J. K. Hale, Ordinary Differential Equations, Wiley-Interscience, New York-London-Sydney, 1969; (revised) Krieger, Malabar, 1980. MR0419901 (54:7918)

[5] L. Hatvani, On the asymptotic stability for a two-dimensional linear nonautonomous differential system, Nonlinear Anal., 25 (1995), 991-1002. MR.1350721 (96k:34105)

[6] D. W. Jordan and P. Smith, Nonlinear Ordinary Differential Equations: An Introduction to Dynamical Systems, 3rd ed., Oxford Texts in Applied and Engineering Mathematics, 2, Oxford University Press, Oxford, 1999. MR1743361 (2000j:34001)

[7] O. Perron, Die Stabilitätsfrage bei Differentialgleichungen, Math. Zeits., 32 (1930), 703-728. MR.1545194

[8] J. Sugie, Convergence of solutions of time-varying linear systems with integrable forcing term, Bull. Austral. Math. Soc., 78 (2008), 445-462. MR2472280 (2009k:34102)

[9] F. Verhulst, Nonlinear Differential Equations and Dynamical Systems, Springer-Verlag, New York-Berlin-Heidelberg, 1990. MR:1036522 (91b:34002)

Department of Mathematics and Computer Science, Shimane University, Matsue 6908504, JAPAN

E-mail address: jsugie@riko.shimane-u.ac.jp

Department of Mathematics and Computer Science, Shimane University, Matsue 6908504, JAPAN

Current address: General Education, Miyakonojo National College of Technology, Miyakonojo 885-8567, Japan

E-mail address: onitsuka@math.shimane-u.ac.jp 